\title{
A double-blind study of the efficacy of apomorphine and its assessment in 'off'-periods in Parkinson's disease
}

\author{
T. van Laar, ${ }^{\mathrm{a}, \mathrm{b}}$, E.N.H. Jansen ${ }^{\mathrm{b}}$, A.W.G. Essink ${ }^{\mathrm{c}}$, C. Neef ${ }^{\mathrm{b}}$, S. Oosterloo ${ }^{\mathrm{d}}$ and R.A.C. Roos ${ }^{\mathrm{a}}$ \\ ${ }^{2}$ Department of Neurology, Leiden University Hospital, Leiden, The Netherlands, ${ }^{b}$ Department of Neurology \& Clinical Pharmacy, \\ Hospital Medisch Spectrum Twente, Enschede, The Netherlands, 'Department of Clinical Pharmacy, Hospital Rijnstate, Amhem, \\ The Netherlands and ${ }^{\mathrm{d}}$ Department of Statistics, Twente University, Enschede, The Netherlands \\ (Received 15 April, 1993) \\ (Revised, received 18 June, 1993) \\ (Accepted 18 June, 1993)
}

Key words: Subcutaneous apomorphine; Parkinson's disease; Columbia-rating scale; Quantitative assessment

\section{Summary}

Five patients with idiopathic Parkinson's disease with severe response fluctuations were selected for a randomized double-blind placebo-controlled study, concerning the clinical effects of subcutaneous apomorphine and its assessment in 'off'-periods. The study was designed as five $n=1$ studies, in which every patient was his own control. The effect of apomorphine was studied by using the Columbia rating scale and quantitative assessments, using tapping, walking and pinboard. There was a significant positive effect of apomorphine, in a mean optimal dose of $2.7 \mathrm{mg}$, with a mean latency of onset of $7.3 \mathrm{~min}$ and a mean duration of response of $96 \mathrm{~min}$. After pretreatment with domperidone, no significant adverse effects were observed. Tapping showed the highest correlation with rigidity and bradykinesia. Walking showed a high correlation with stability and gait. Pinboard testing did not give additional information. The first conclusion was that apomorphine proved to be a significantly effective dopamine agonist, proven now also by a double blind placebocontrolled study. Secondly it was concluded that assessment of clinical effect in parkinsonian patients can be performed best by combining the Columbia item tremor with tapping and walking scores.

\section{Introduction}

The incidence of disabling 'on-off' fluctuations in Parkinson's disease (PD) increases with the duration of levodopa treatment. After 10 years of sustained therapy, most patients are affected [1]. Once established, severe motor fluctuations are difficult to manage with changes in prescription strategies, e.g. dose-fractioning, sustained-release medication and dopamine agonists. Since 1987, apomorphine, a potent directly acting dopamine agonist, has been introduced as a subcutaneous injection

Correspondence to: T. van Laar, MD, Department of Neurology, Leiden University Hospital, Rijnsburgerweg 10, P.O. Box 9600, 2300 RC Leiden, The Netherlands. Tel.: 071-262111. therapy in PD [2]. Many authors have published the posive effects of subcutaneous apomorphine, in reversing the "off'-period motor deficits [3-6]. None of these studies, however, used a placebo-controlled design and most studies had a short follow-up, in which placebo effects could not be excluded. Placebo effects can not be ignored, as was shown in the study with the dopamine agonist pergolide, by Olanow et al. [7].

There is only one previous report of a placebo-controlled study with apomorphine [8]. In this study with 8 patients, all patients only received $1 \mathrm{mg}$ apomorphine, without domperidone pretreatment and whilst taking their usual medication. Therefore, we did a randomized double-blind placebo-controlled study in 5 patients, to evaluate the statistical significance of the clinical benefit 
of subcutaneous administered apomorphine. We also considered the correlation between clinical and quantitative assessments of these patients. Pharmacokinetic parameters and their relation to the clinical efficacy of apomorphine have also been studied.

\section{Patients and methods}

Five patients, two women and three men, with idiopathic PD, mean age of 54.2 years (range 29-68 years) and a mean duration of PD of 12.4 years (range 7-23 years), were selected for this study. One patient already showed parkinsonian symptoms at the age of 22 . However, extensive laboratory analysis and CT-scanning of the brain did not reveal any abnormality. Therefore this patient also was included in this study. Three patients were in Hoehn and Yahr stage IV and two patients in stage III. They had used levodopa medication with a peripheral decarboxylase inhibitor for 9.6 years (range 2 20 years). All patients had had response fluctuations for at least half a year. Their anti-parkinsonian medication was kept unchanged for at least 1 month prior to the study. All conventional methods to improve their response fluctuations had failed.

Patients with other neurological or general internal diseases were excluded, as well as patients with a mini mental state examination (MMSE) of less than 24 points. All patients gave informed consent and the study was approved by the Medical Ethical Committee of the hospital. The patients were admitted for one week during the study period. Before and after the study, each patient underwent physical examination, routine laboratory investigation and ECG registration. To avoid adverse effects of apomorphine, domperidone $20 \mathrm{mg}$ t.i.d. was given. All patients were titrated up to an optimal dose of subcutaneous apomorphine $\mathrm{HCl}(1 \%, 10 \mathrm{mg} / \mathrm{ml})$, starting with $1 \mathrm{mg}$, with subsequent $1 \mathrm{mg}$ increments. The maximum dose was $10 \mathrm{mg}$. Optimal dose was defined as the maximum tolerated dose, with the best motor response in combination with minimal adverse effects. In the next ten 'off'-periods, with intervals of at least $3 \mathrm{~h}$, apomorphine or placebo $(0.9 \%$ sodiumhydrochloride solution) was injected with a non-transparent injection pen, following a randomization table, of which the key was kept by the pharmacist. Each patient was his own control in these five $n=1$ studies.

Before and at the time of best motor response, or $0.5 \mathrm{~h}$ after the injection, the following assessments were made: the Columbia rating, a finger/foot-tapping score during $30 \mathrm{~s}$, a timed walking over $25 \mathrm{~m}$ and a timed pinboard test, as well as the blood pressure. The Columbia rating has 5 items: tremor, rigidity, gait, bradykinesia and stability, each scored according to a 5-point scale. The exact one-tailed significance probabilities $(p)$ of the mean difference-scores of the separate items of the Columbia rating scale and the quantitative assessments, were computed by using the Mann-Whitney test. Also the $p$-values

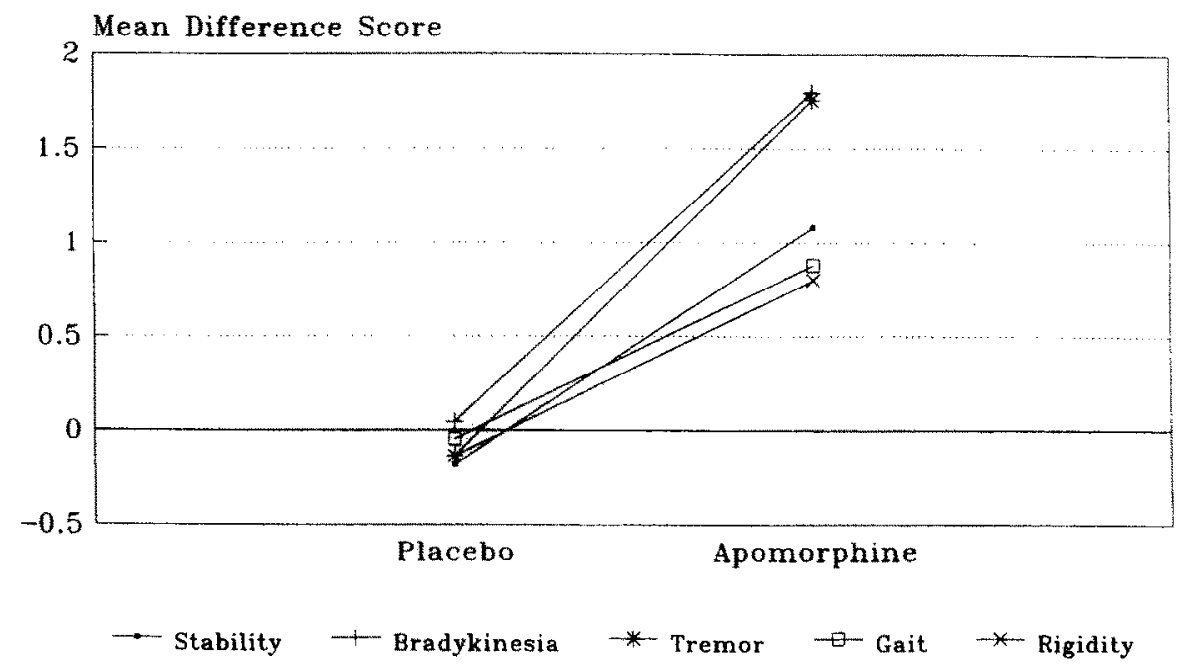

for every item the combined Mann-Whitney statistic yields $p<.001$ (see text)

Fig. 1. Separate Columbia items. Mean difference scores of the separate Columbia items. For every item the combined Mann-Whitney statistic yields $P<0.001$. 
for the summed Columbia items were computed, for each separate patient. The difference scores of the separate quantitative variables were summed. The difference scores of walking and pin-board were turned from negative into positive, because a shorter time means an improved result. Each quantitative variable was standardized (on mean 0 and standard deviation 1) over all trials, in order to obtain a sum score to which each of the variables contributes approximately equally. Finally, the sum score was standardized again and written down the yaxis. To get an idea of the most informative combination of Columbia items and quantitative assessments, a Spearman correlation coefficient was computed for all combinations. All assessments were made by one author, to eliminate inter-observer variabilities. The same author did the run-in of optimal dose finding injections. The latency of onset and duration of response were recorded, as well as possible adverse effects.

From each patient 2 time-concentration profiles of serum-concentration apomorphine were made in the dose-finding series. The first data set was obtained after injecting the starting dose of $1 \mathrm{mg}$ and the second after giving the optimal dose of apomorphine. After injecting $1 \mathrm{mg}$, blood samples were taken according a fixed timeschedule $t=0,3,6,9,12,15,30,45,60$ and $120 \mathrm{~min}$, by means of an intravenous catheter. The samples were stored at $-20^{\circ} \mathrm{C}$ until analysis by HPLC [9].

After injection of the optimal dose, 3 samples, only around the $T_{\max }$ from the $1 \mathrm{mg}$-profile, were taken and analyzed. The presence of apomorphine in the blood samples was regarded as a control of the randomisation table.

\section{Results}

The mean optimal dose of apomorphine was $2.7 \mathrm{mg}$ with a range of $1-5 \mathrm{mg}$. All 5 patients responded very well to apomorphine. All patients reported a sudden relief of their 'off'-symptoms, often accompanied with yawning. The latency of onset had a mean value of 7.3 min (range 1.5-15 min). The mean duration of response was $96 \mathrm{~min}$ (range 20-120 min). In computing the following data, 3 out of 50 trials ( 5 patients with 10 trials cach) were excluded, because the contents of the injection pen, according to the list of the pharmacist, did not correspond with the HPLC-analysis of the serum levels. The mean difference scores of the separate Columbia items showed a significant improvement of all items, after apomorphine. For every item the combined Mann-Whitney statistic yields $p<0.001$ (Fig. 1). The most significant scores were reached with the items tremor and stability, with Z-values ranging from 4.45-4.83.

The mean difference scores of the sum of Columbiaitems for each patient are shown in Fig. 2. Only one patient did not show a significant improvement of the total Columbia score after apomorphine. The other patients showed $p$-values ranging from $0.00-0.03$. The combined

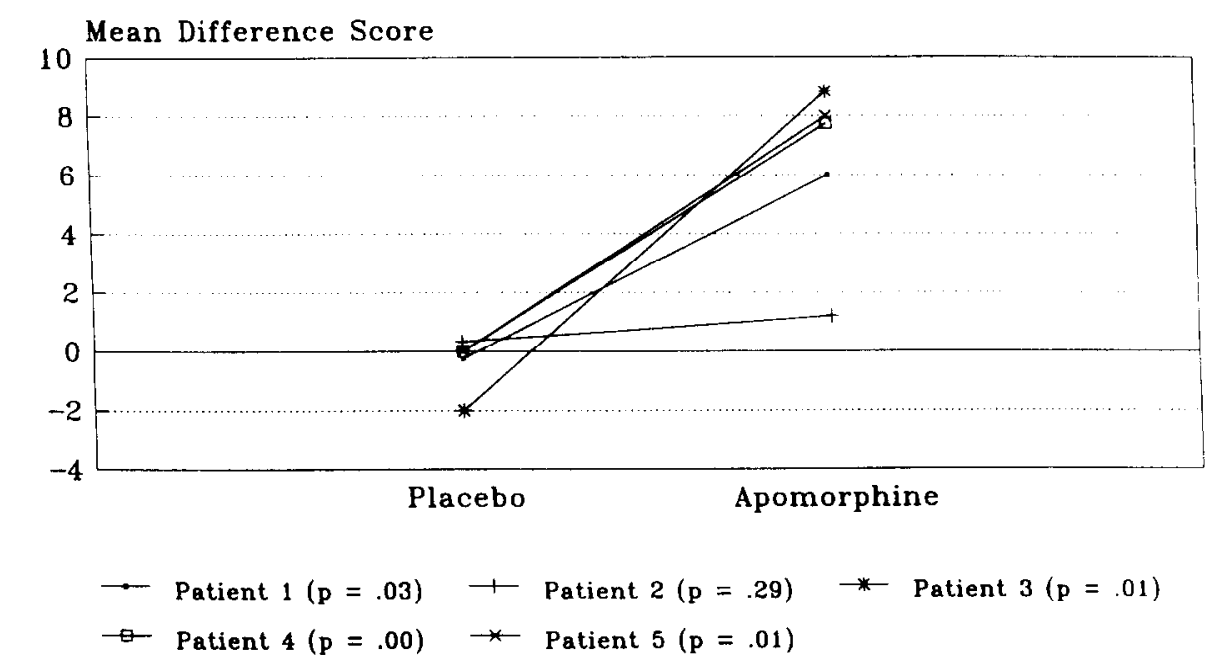

Combined Mann-Whitney test (see text):

$\mathrm{Z}=4.76, \mathrm{p}=.001$

Fig. 2. Sum of Columbia items. Mean difference scores of the sum of Columbia items. The results of each separate patient are shown. 


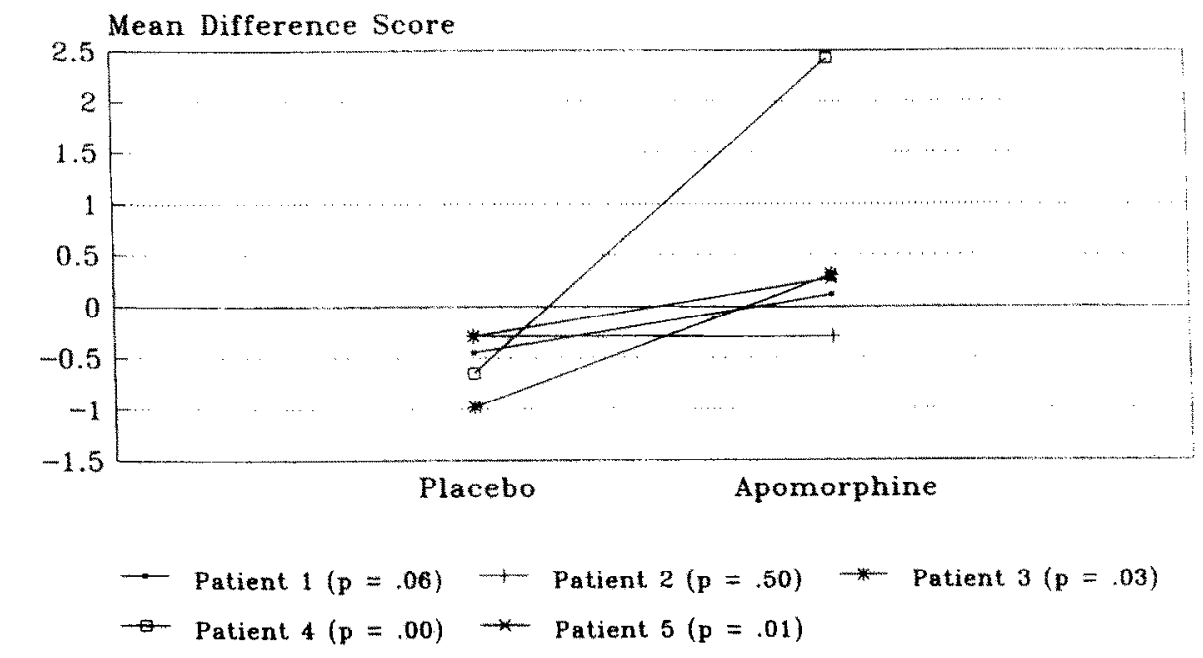

Combined Mann-Whitney test (see text):

$\mathrm{Z}=3.79, \mathrm{p}=.001$

Fig. 3. Sum of quantitative variables. Mean difference scores of the sum of quantitative variables. The results of each separate patient are shown.

Mann-Whitney test statistic, in which the results of all patients are put together, yielded $Z=4.76$, which resulted in $p=0.001$. This means an overall statistically significant positive effect of apomorphine. When analyzed separately, the mean difference in the quantitative sum scores were not significant better in 2 patients with $p=0.06$ and $p=0.50$ (Fig. 3). After summing the quantitative variables (pinboard, tapping and walking) of all patients, however, there was a significant improvement $(p=0.001)$ after apomorphine vs. placebo, with $Z=3.79$.

The correlations, computed with the Spearman test, between the Columbia items and the quantitative items showed some interesting results (Table 1). Tapping showed the best correlations with the Columbia items. The coefficients ranged from $0.65-0.81$, with $p<0.001$. From these items, especially bradykinesia and rigidity correlated very well with tapping. The other items did not add much, looking at the correlation coefficient with the total Columbia score $(0.84)$. Walking showed the highest correlation with gait and stability, as one should have expected (correlation coefficients 0.76 and 0.74 respectivily, $p<0.001$ ). Pinboard testing did not show an important correlation with one of the Columbia items.

Very few adverse effects were reported. Only one patient showed nausea, without vomiting. Four patients showed dyskinesia, in combination with the maximal response. One patient had short-lasting twinkling in both legs. Also one patient had a very short-lasting worsening of tremor and another patient complained about a feeling of warmth and perspiration, both just before the onset of clinical effect.

Two patients spontaneously reported a lower level of motor functioning at the end of clinical effect. as compared to their basic level before the test. There was no influence on blood pressure. Routine laboratory investigation, ECG and physical examination revealed no abnormalities. The data obtained from the time-concentration figures show a mean $T_{\max }$ of 10 min (range 7.5-13 min) and a mean $C_{\max }$ of $22 \mathrm{ng} / \mathrm{ml}$ (range $12-43 \mathrm{ng} / \mathrm{ml}$ ).

\section{Discussion}

In this study we proved that apomorphine is statistically significantly more efficacious, compared to placebo, in 'off'-periods in PD. This was shown in the clinical scoring, as well in the quantitative data. The mean optimal dose of apomorphine injection was $2.7 \mathrm{mg}$. which is comparable to data already published by several authors [4-6]. The same holds true for the latency of onset of $7.3 \mathrm{~min}$. Our duration of response $(96 \mathrm{~min})$ is slightly longer than in most other studies. Only Poewe reported a duration of response varying from $1.0-2.5 \mathrm{~h}$, after one single injection with apomorphine [10]. A very interesting phenomenon is the worsening at the end of motor response and just before onset of clinical effect. which might be explained by an inhibitory feedback ac- 
TABLE 1

SPEARMAN CORRELATIONS

\begin{tabular}{llll}
\hline & Tapping & Walking & Pinboard \\
\hline Rigidity & 0.76 & 0.53 & 0.52 \\
Tremor & 0.73 & 0.54 & 0.46 \\
Bradykinesia & 0.81 & 0.60 & 0.55 \\
Stability & 0.68 & 0.74 & 0.49 \\
Gait & 0.65 & 0.76 & 0.50 \\
Total Columbia & 0.84 & 0.68 & 0.53 \\
\hline
\end{tabular}

$P<0.001$ for all correlation coefficients.

tion of apomorphine on the pre-synaptic dopamine receptor $[11,12]$.

In this study, tapping showed the highest correlation with especially rigidity and bradykinesia and walking showed a high correlation with stability and gait. Pinboard testing did not add important information. This suggests a possibility to reduce our qualitative and quantitative assessments, without losing significant information. From other studies the reliability of the Columbia items, with great interobserver agreements, was established [13]. Therefore, based on our results, a combination of the Columbia item tremor, which can be scored in a short time, combined with tapping and walking scores, gives the best information.

The adverse effects after pretreatment with domperidone were very mild and not disabling. This is a great advantage as compared to other dopamine agonists such as lisuride and bromocriptine, which produce more psychiatric and cardiovascular complications [7,14]. One of the possible explanations is the anti-psychotic property of the piperidine side-chain of apomorphine. The other possible adverse effects of apomorphine can succesfully be blocked by domperidone.

Finally, our results show that apomorphine is a significantly effective tool in the management of PD, supported by the experiences of many neurologists, but hardly proven before.

Also after long-term treatment (1-3 years) with apomorphine, a significant efficacy can be maintained in almost all patients. Only the optimal dose slightly increases and the duration of effect slightly decreases during years of treatment. The most important limiting factor for the long-term use of subcutaneous apomorphine sometimes is the local irritability, leading to e.g. subcutaneous nodules. These nodules are not harmful, when treated with
$1 \%$ hydrocortisone creme and/or hyaluron acid (lasonil), but give sometimes cosmetic problems. In our experience therefore apomorphine deserves an important role in the treatment of response fluctuations in Parkinson`s disease.

\section{References}

1 Lees, A.J. The on-off phenomenon. I. Neurol. Neurosci. Psychiatry 1989: 29-37.

2 Stibe, C.M.H., Lees, A.J. and Stern, G.M. Subcutaneous infusion of apomorphine and lisuride in the treatment of Parkinsonian on-off fluctuations. Lancet 1987: 871 .

3 Cotzias, G.C., Papavasiliou, P.S., Tolosa, E.S. et al. Treatment of Parkinson's disease with apomorphines. N. Engl. J. Med. 1976; 294: 567-723.

4 Pollak, P., Champay, A.S., Hommel, M. et al. Subcutaneous apomorphine in Parkinson's disease. J. Neurol. Neurosci. Psychiatry 1989; 52: 544.

5 Frankel, J.P., Lees, A.J., Kempster, P.A. and Stern, G.M. Subcutaneous apomorphine in the treatment of Parkinson's disease. J. Neurol. Neurosci. Psychiatry 1990; 53: 96-101.

6 Stibe, C.M.H., Kempster, P.A., Lees, A.J. and Stern, G.M. Subcutaneous apomorphine in Parkinsonian on-off oscillations. Lancet 1988: 403-406.

7 Olanow, C.W. and Alberts, M.J. Double-blind controlled study of pergolide mesylate in the treatment of Parkinson's disease. Clin. Neuropharmacol. 1987; 10: 178-185, Raven Press, New York.

8 Hardie, R.J., Lees, A.J. and Stern, G.M. On-off fluctuations in Parkinson's disease. Brain 1984; 107: 487-506.

9 Essink, A.W.G., Loohuis, C.P.G.G., Klein Elhorst, J.T. et al. Selective and quantitative isolation and determination of apomorphine in human plasma. J. Chrom. Biomed. Appl. 1991; 570; 419-424.

10 Poewe, W., Kleedorfer, B., Gerstenbrand, F. and Oertel, W.II. Die Behandlung von Parkinsonpatienten mit L-dopa Wirkungsfluktuation mittels subcutanen Apomorphingaben. Akt. Neurol. 1989; 16: 73-77.

11 Hughes, A.J., Lees, A.J. and Stern, G.M. The motor response to sequential apomorphine in Parkinsonian fluctuations. J. Neurol. Neurosci. Psychiatry 1991: 54: 358-360.

12 Nutt, J.G., Gancher, S.T. and Woodward, W.R. Does an inhibitory action of levodopa contribute to motor fluctuations? Neurology 1988; 38: 1553-1557.

13 Montgomery, G.K., Reynolds, N.C. and Warren, R.M. Qualitative assessment of Parkinson's disease: study of reliability and data reduction with an abbreviated Columbia scale. Clin. Neuropharmacol. 1985; 8: 83-92, Raven Press, New York.

14 Ruggieri, S., Stocchi, F., Carta, A. et al. Side effects of subcutaneous apomorphine in Parkinson's disease. Lancet 1989: 566 . 